\title{
The Energy Expenditure of Free-Living Physical Activities in Primary Schoolchildren
}

\begin{abstract}
Alison L. Innerd and Liane B. Azevedo
Background: The aim of this study is to establish the energy expenditure (EE) of a range of child-relevant activities and to compare different methods of estimating activity MET. Methods: 27 children (17 boys) aged 9 to 11 years participated. Participants were randomly assigned to 1 of 2 routines of 6 activities ranging from sedentary to vigorous intensity. Indirect calorimetry was used to estimate resting and physical activity EE. Activity metabolic equivalent (MET) was determined using individual resting metabolic rate (RMR), the Harrell-MET and the Schofield equation. Results: Activity EE ranges from 123.7 $\pm 35.7 \mathrm{~J} /$ $\mathrm{min} / \mathrm{Kg}$ (playing cards) to $823.1 \pm 177.8 \mathrm{~J} / \mathrm{min} / \mathrm{kg}$ (basketball). Individual RMR, the Harrell-MET and the Schofield equation MET prediction were relatively similar at light and moderate but not at vigorous intensity. Schofield equation provided a better comparison with the Compendium of Energy Expenditure for Youth. Conclusion: This information might be advantageous to support the development of a new Compendium of Energy Expenditure for Youth.
\end{abstract}

Keywords: indirect calorimetry, METs, playground, individual RMR

A Compendium of Energy Expenditure for Youth was developed in 2008 with a list of over 200 activities that are usually performed by children and adolescents. ${ }^{1}$ Only $35 \%$ of the activities reported in the Compendium were based on activity data measured in youth, while the remaining were estimated by Compendium of Physical Activity in Adults. ${ }^{2}$ However, the resting energy expenditure (EE) for adults is lower than children and although activity EE could also be lower in adults, the metabolic equivalent (MET) is slightly higher compared with children. ${ }^{3}$ Likewise, EE in children can vary according to pubertal status. ${ }^{3}$ Furthermore, the MET values estimated in Compendium used predicted (Schofield equation) rather than measured resting metabolic rate (RMR) to calculate MET. ${ }^{2}$

There is a need for an update to the Compendium of Energy Expenditure for Youth with accurate and direct measurements of physical activity from different ages. Therefore, the aim of this study was to provide information on EE of a range of playground and child-relevant activities in schoolchildren aged between 9 to 11 years old. The secondary aim was to compare the MET of these activities using different estimated methods including: individually measured RMR, the Harrell-MET, ${ }^{3}$ the Schofield equation and the previously established MET from the Compendium of Energy Expenditure for Youth. ${ }^{1}$

\section{Methods}

\section{Ethical Approval}

This study was approved by Teesside University, School of Health and Social Care Research and Governance Ethics Committee (protocol number: 056/13). Written informed consent was obtained

Innerd (a.innerd@tees.ac.uk) is with the School of Social Sciences, Business, and Law; Azevedo is with the Health and Social Care Institute, Teesside University, United Kingdom. from the Head Teacher and parental/guardian of the participating children as well as child assent before the study.

\section{Participants}

A total of 27 (10 girls, 17 boys) schoolchildren aged 9 to 11 from 1 primary school in the North East of England participated in the study.

\section{Study Design}

All testing procedures were conducted at the school. The testing consisted of 2 phases (separated by at least a day): 1) resting EE and 2) physical activities. To test as many activities as possible there were 2 different physical activity routines. The physical activities were selected based on common reported activities of schoolchildren within the North East of England. ${ }^{4}$

\section{Measurements}

Height $(\mathrm{cm})$ and weight $(\mathrm{kg})$ were measured before testing. Children had to wear light clothing and removed shoes. Height was measured to the nearest $0.1 \mathrm{~cm}$ using a portable stadiometer (Leicester Height Measure, Child Growth Foundation, London, United Kingdom). Weight was measured to the nearest $0.1 \mathrm{~kg}$ using calibrated scales (Seca 761, Seca Weighing and Measuring Systems, Birmingham, England).

\section{Indirect Calorimetry}

Before each test, the oxygen and carbon dioxide analyzers and the flow turbine were calibrated according to the manufacturer's instructions for the Cosmed K4b2. The child's information [height, mass (plus $2 \mathrm{~kg}$ - to account for the weight of the Cosmed and shoes) and age] was inputted into the Cosmed software before testing. The children wore the Cosmed K4b2 for the duration of the resting measures and physical activity routine. The indirect calorimeter measured expired gases on a breath-by-breath basis. 


\section{Resting Energy Expenditure}

The initial stage of the testing was the estimation of resting EE. Resting measures were taken on a different day to the physical activity trials. The children were informed to fast for a minimum of 2 hours before their respective test and were asked to avoid vigorous intensity activity 24 hour before testing. The testing was conducted in a quiet, darkened room at the school and distractions were prevented as much as possible. The children attached the heart rate monitor, and the face mask was placed for habituation for 5 to 10 minutes. The participants were told to lay comfortably in a supine position, on a mat with a pillow to rest the head. RMR was measured for 12 min which appears to be an acceptable duration for practical purposes. ${ }^{5}$

\section{Protocol}

Children were randomly allocated to 1 of 2 routines by 'names into a hat' method. Table 1 displays a description of the activities performed in the 2 routines. Both routines consisted of 2 low-intensity, 2 moderate and 2 vigorous-intensity activities following the classifications in the Compendium of Energy Expenditure for Youth. ${ }^{1}$

The activities were performed for 5 minutes, followed by 5 minutes rest between each activity. We determined that the child had recovered once the HR was within $10 \%$ of the resting HR. All activities were performed standing, apart from playing cards, drawing and watching TV. To motivate and maintain the activity level, a member of the research team participated in the activities that involve team participation (soccer, tag and basketball). However, children were informed to conduct the activities at their own pace, apart from walking which was controlled by a metronome. For the running activity, SmartSpeed timing gates (SmartSpeed, Fushion Sport, United Kingdom) were placed in a $15 \mathrm{~m}$ square area and 2 children performed the activity at the same time. Each child was given a light to follow, when the light flashed, the child had to run and break the beam, this continued over a 5-minute period. The children were encouraged to break as many beams as possible but maintain a steady pace to complete the 5 minutes of activity. The light sequence was random to replicate the sporadic nature of running. $\mathrm{VO}_{2}$ and $\mathrm{VCO}_{2}$ were monitored continuously throughout all activities using the Cosmed and the researcher recorded the exact time of each activity and marked the event button on the Cosmed unit.

\section{Data Analysis}

Data were coded and downloaded using the respective software package for analysis. To calculate the individual resting value the data were reduced to mid- 5 minutes by deleting the first 2 minutes and last 3 minutes of data. Resting EE was calculated as an average across the remaining of $5 \mathrm{~min}$. EE was calculated using the Weir equation. ${ }^{6}$

The physical activity data were trimmed from 5 minutes to 2 minutes and 25 seconds by deleting the first 2 minutes and the last 15 seconds of data. This was necessary to remove the initial activity period (2-min) when the child had not reached steady state and the final 15 -sec when the activity was terminating. Data were also filtered so that extreme outliers (data with more than 3 standard deviations from the mean) caused by measurement error were deleted. Once this editing stage had been completed the mean $\mathrm{VO}_{2} /$ $\mathrm{kg}, \mathrm{EE}(\mathrm{J} / \mathrm{kg} / \mathrm{min})$ and MET values were calculated for each physical activity. MET values were determined by 1 ) dividing $\mathrm{VO}_{2} / \mathrm{kg}$ by the individual metabolic resting value; 2$)$ dividing $\mathrm{VO}_{2} / \mathrm{kg}$ by $5.92(\mathrm{ml} /$ min $/ \mathrm{kg}$ ), the Harrell-MET; ${ }^{3}$ and 3) Schofield predicted RMR. The coefficient of variance of $\mathrm{EE}(\mathrm{J} / \mathrm{min} / \mathrm{kg})$ of activities was calculated by dividing the standard deviation by the mean.

\section{Results}

We recorded data from 32 participants, however we excluded all data from 5 participants due to equipment failure and measurement errors $(\mathrm{N}=27)$. The mean $(\mathrm{SD})$ age $(\mathrm{y})$, height $(\mathrm{cm})$, weight $(\mathrm{kg})$ and body mass index (BMI) of all participants was $10.3(0.6) \mathrm{y}, 146.4$ $(6.2) \mathrm{cm}, 38.2(7.9) \mathrm{kg}$ and $17.6(3) \mathrm{kg} / \mathrm{m}^{2}$, respectively. According to the British growth reference (1990), $82 \%$ of the participants were classified as healthy weight (2nd-85th centile), $11 \%$ were classified as overweight ( 85 th -95 th centile) and $7 \%$ were classified as obese ( $\geq 95$ th centile). Although girls had similar height than boys (146.2 $\mathrm{cm}$ girls vs. $146.5 \mathrm{~cm}$ boys), girls weight and BMI were higher than boys (weight: $39.9 \mathrm{~kg}$ girls vs. $37.2 \mathrm{~kg}$ boys; BMI: 18.5 girls vs. 17.1 boys).

\section{Resting Metabolic Rate}

The mean results for the total sample and for each sex are shown in Table 2.

The average resting $\mathrm{VO}_{2}$ was $6.3\left(\mathrm{ml} \cdot \mathrm{min} \cdot{ }^{-1} \cdot \mathrm{kg}^{-1}\right)$, with the boys having a slightly higher $\mathrm{VO}_{2}$ than the girls. The mean absolute resting $\mathrm{EE}(\mathrm{kcal} \cdot \mathrm{min})$ was $1.14(\mathrm{kcal} \cdot \mathrm{min})$ with the boys displaying lower EE than the girls.

\section{Activity Energy Expenditure}

Fourteen participants performed routine 1 and 13 participants performed routine 2 (Table 1). As shown in Table 3, the activity with the lowest EE (123.7 J·min. $\mathrm{kg}$ ) was playing cards and the activity with the highest EE was basketball $(823.1 \mathrm{~J} \cdot \mathrm{min} \cdot \mathrm{kg})$. The MET values derived using the Compendium of Energy Expenditure in Youth $^{1}$ are higher for most activities than the MET values derived from individual RMR or Harrell-MET. The discrepancy between the MET values appears to increase as the intensity of the physical activities increases. The MET values using the Schofield equation at vigorous intensity matches more closely to the Compendium. Table 3 also presents the interindividual variability in all activities.

\section{Discussion}

The study provides information of direct measurement energy costs in different playground and free-living activities in children aged 9 to 11 years old. When comparing the 3 methods of calculating METs (individual, Harrell-MET and Schofield), the predicting values at light and moderate intensity activities were fairly similar. However for activities above 5 MET, the individual MET and Harrell-MET appeared to underestimate the value when compared with Schofield and the Compendium of Energy Expenditure for Youth. The similarity of individual RMR and Harrell-MET equation on MET values throughout the range of activity intensities suggests that Harrell-MET equation may be a suitable option when measuring individual RMR is not possible. Schofield equation provided a better comparison in general with the Compendium of Energy Expenditure for Youth.

The main strengths of the study are the use of direct measurements of EE at rest and during the activities, the range in intensity of activities performed and the mixed weight population (18\% overweight or obese). However, the sample size was small; 


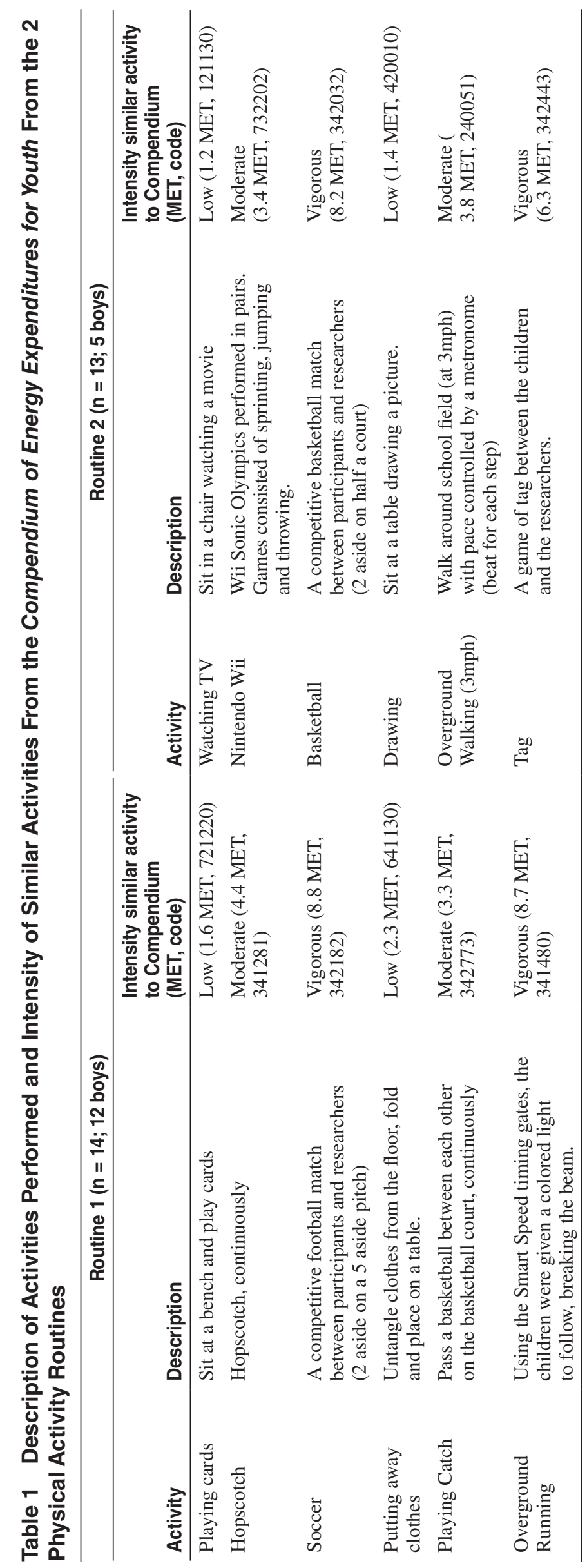


Table 2 Mean, Standard Deviation and Confidence Interval (Cl) of Resting Values in $\mathrm{VO}_{2}(\mathrm{ml} / \mathrm{min} / \mathrm{kg})$ and Energy Expenditure $(\mathrm{kcal} / \mathrm{min})$

\begin{tabular}{|c|c|c|c|c|c|c|}
\hline & \multicolumn{3}{|c|}{$\mathrm{VO}_{2}(\mathrm{ml} / \mathrm{min} / \mathrm{kg})$} & \multicolumn{3}{|c|}{ Energy expenditure (kcal/min) } \\
\hline & Mean & SD & $95 \% \mathrm{Cl}$ & Mean & SD & $95 \% \mathrm{Cl}$ \\
\hline Total & 6.26 & 0.94 & $5.90-6.62$ & 1.14 & 0.27 & $1.03-1.24$ \\
\hline Boys & 6.39 & 1.00 & $5.92-6.86$ & 1.12 & 0.23 & $1.01-1.23$ \\
\hline Girls & 6.04 & 0.85 & $5.51-6.57$ & 1.16 & 0.35 & $0.94-1.38$ \\
\hline
\end{tabular}

Table 3 Estimated Energy Expenditure (J/min/ $/ \mathrm{kg}$ ) and METs for Each Activity (Mean \pm SD) Estimated Using the Individual RMR, the Harrell-MET, Schofield Equation and the Predefined METs From Similar Activities From the Compendium of Energy Expenditures for Youth

\begin{tabular}{|c|c|c|c|c|c|c|}
\hline Activity & $\begin{array}{c}\text { Energy } \\
\text { expenditure }\end{array}$ & $\begin{array}{c}\text { METs } \\
\text { individual RMR }\end{array}$ & $\begin{array}{c}\text { METs } \\
\text { Harrell-MET }\end{array}$ & $\begin{array}{c}\text { METs } \\
\text { Schofield }\end{array}$ & $\begin{array}{c}\text { METs } \\
\text { compendium }\end{array}$ & $\begin{array}{c}\text { Energy expenditure } \\
\text { coefficient } \\
\text { of variance (\%) }\end{array}$ \\
\hline Watching TV & $129.9 \pm 35.8$ & $1.1 \pm 0.3$ & $1.1 \pm 0.3$ & $1.3 \pm 0.4$ & 1.2 & 27.6 \\
\hline Drawing & $149.1 \pm 33.2$ & $1.2 \pm 0.3$ & $1.3 \pm 0.3$ & $1.5 \pm 0.3$ & 1.4 & 22.2 \\
\hline Playing Cards & $123.7 \pm 35.7$ & $1.0 \pm 0.3$ & $1.0 \pm 0.3$ & $1.2 \pm 0.3$ & 1.6 & 28.9 \\
\hline Putting away clothes & $328.6 \pm 51.8$ & $2.7 \pm 0.7$ & $2.7 \pm 0.4$ & $3.3 \pm 0.5$ & 2.3 & 15.8 \\
\hline Nintendo Wii & $243.7 \pm 69.2$ & $2.0 \pm 0.7$ & $2.1 \pm 0.6$ & $2.5 \pm 0.8$ & 3.4 & 28.4 \\
\hline Walking & $334.6 \pm 59.5$ & $2.7 \pm 0.5$ & $2.9 \pm 0.5$ & $3.4 \pm 0.6$ & $3.8^{*}$ & 17.8 \\
\hline Playing Catch & $492.3 \pm 139.1$ & $4 \pm 1.1$ & $4.2 \pm 1.2$ & $4.9 \pm 1.2$ & 3.3 & 28.2 \\
\hline Hopscotch & $510.6 \pm 162.3$ & $4.2 \pm 1.5$ & $4.3 \pm 1.4$ & $5.1 \pm 1.5$ & 4.4 & 31.8 \\
\hline Tag & $675 \pm 108.7$ & $5.5 \pm 1.6$ & $5.7 \pm 1.3$ & $6.7 \pm 1.4$ & 6.3 & 16.1 \\
\hline Soccer & $803.4 \pm 139.5$ & $6.6 \pm 1.4$ & $6.6 \pm 1.1$ & $8.0 \pm 1.2$ & 8.8 & 17.4 \\
\hline Basketball & $823.1 \pm 177.8$ & $6.7 \pm 1.7$ & $6.8 \pm 1.1$ & $8.2 \pm 1.3$ & 8.2 & 21.6 \\
\hline Running & $762.6 \pm 198.8$ & $6.2 \pm 1.7$ & $6.3 \pm 1.3$ & $7.5 \pm 1.8$ & $8.7 * *$ & 26.1 \\
\hline
\end{tabular}

Abbreviations: RMR, resting metabolic rate.

* MET from walking was calculated using the regression equation ${ }^{2}$ at the walking speed of $1.34 \mathrm{~m} \cdot \mathrm{s}^{-1}$ for a $10-y e a r-o l d ~ c h i l d$.

** MET from running was calculated using the regression equation ${ }^{2}$ at the running speed of $2.92 \mathrm{~m} \cdot \mathrm{s}^{-1}$ for a 10 -year-old child.

therefore future studies with larger samples would be advantageous. Likewise, we did not measure the different stage of maturation which could affect EE. ${ }^{3}$ Similarly, RMR was performed at school and not in a laboratory environment. Although we tried to control for light and noise there could be distractions in the environment that might have elevated children's RMR. RMR has been previously reported as $5.92 \mathrm{ml} / \mathrm{kg} / \mathrm{min}(95 \% \text { CI: } 5.67-6.17)^{3}$ in a large sample of children $(\mathrm{N}=114)$ of the same age in a controlled laboratory environment. This RMR is considerably lower than the average value reported here $6.26 \mathrm{ml} / \mathrm{kg} / \mathrm{min}$ (95\% CI: 5.90-6.62), which might, as a consequence, have underestimated the MET values of the activities. However, RMR appears to vary substantially according to the resting protocol applied. In the current study we used similar resting protocol as a previous study ${ }^{7}$ (ie, 2-h fasting). Although the previous study ${ }^{7}$ was performed in a laboratory environment, their reported value $(1.4 \mathrm{kcal} / \mathrm{min}, 95 \% \mathrm{CI}$ : $1.03-2.10)$ was within the CI limits of our study (Table 2).

There was moderate interindividual variability within the different activities. Some activities such as putting clothes away and walking presented lower variability (16\% and $18 \%$ respectively) while activities such as hopscotch presented high variability (32\%). This might reflect the nature of the activity in relation to variation in movement effort from each participant. However, this variability might also be inflated due the small sample size. Similar to our study, a previous study ${ }^{8}$ found no relationship between the intensity of the activity and the $\mathrm{CV}$ as higher $\mathrm{CV}$ was observed for lower intensity activities (eg, playing computer games) and vigorous activities (eg, biking).

This study provides an accurate estimation of the energy costs of a variety of commonly performed, child-relevant physical activities within a field-based setting, and also the associated MET values for the each activity. This information might be advantageous to support the development of a new Compendium of Energy Expenditure for Youth.

\section{Acknowledgments}

The authors would like to thank the primary school and the schoolchildren for participating in the study. Also Christine Steel and Katy Weston for assisting with the data collection.

\section{References}

1. Ridley K, Ainsworth BE, Olds TS. Development of a compendium of energy expenditures for youth. Int J Behav Nutr Phys Act. 2008;5(1):45. PubMed doi:10.1186/1479-5868-5-45 
2. Ainsworth B. E, Haskell W. L, Whitt M. C, et al. Compendium of physical activities: an update of activity codes and MET intensities. Med Sci Sports Exerc. 2000;32(9; SUPP/1):S498-S504. doi:10.1097/00005768-200009001-00009

3. Harrell JS, McMurray RG, Baggett CD, et al. Energy costs of physical activities in children and adolescents. Med Sci Sports Exerc. 2005;37:329-336. PubMed doi:10.1249/01. MSS.0000153115.33762.3F

4. Moore HJ, Ells LJ, McLure SA, et al. The development and evaluation of a novel computer program to assess previous-day dietary and physical activity behaviours in school children: The Synchronised Nutrition and Activity Program (SNAP). Br J Nutr. 2008;99:1266-1274. PubMed doi:10.1017/S0007114507862428
5. Ventham JC, Reilly JJ. Reproducibility of resting metabolic rate measurement in children. Br J Nutr. 1999;81(6):435-437. PubMed doi:10.1017/S0007114599000781

6. Weir JB. New methods for calculating metabolic rate with special reference to protein metabolism. J Physio. 1949;109(1-2):1-9. PubMed doi:10.1113/jphysiol.1949.sp004363

7. Corder K, Brage S, Wareham NJ, et al. Comparison of PAEE from combined and separate heart rate and movement models in children. Med Sci Sports Exerc. 2005;37(10):1761-1767. PubMed doi:10.1249/01.mss.0000176466.78408.cc

8. Pfeiffer KA, Schmitz KH, McMurray RG, et al. Physical activities in adolescent girls: variability in energy expenditure. Am J Prev Med. 2006;31(4):328-331. PubMed doi:10.1016/j.amepre.2006.06.002 